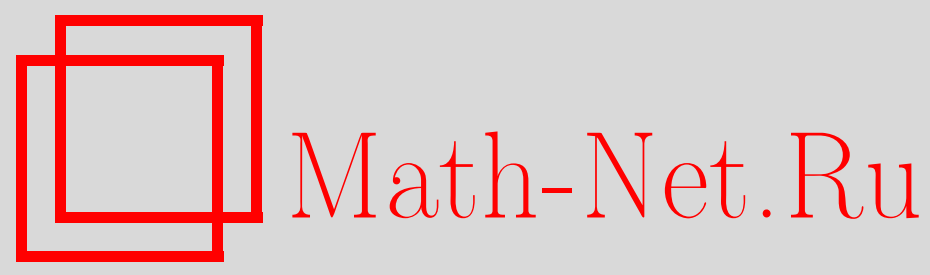

И. Х. Беккер, О смешанных абелевых группах с периодическими группами автоморфизмов, Матем. заметки, 1997, том 61, выпуск 4, 483-493

DOI: https://doi.org/10.4213/mzm1528

Использование Общероссийского математического портала Math-Net.Ru подразумевает, что вы прочитали и согласны с пользовательским соглашением http://www . mathnet.ru/rus/agreement

Параметры загрузки:

IP : 54.209 .52 .79

26 апреля 2023 г., 13:19:30

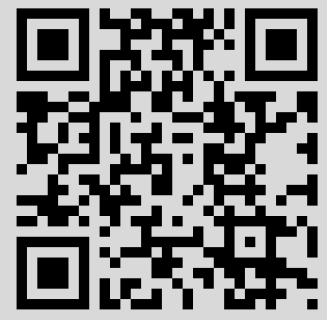




\title{
О СМЕШАННЫХ АБЕЛЕВЫХ ГРУППАХ \\ С ПЕРИОДИЧЕСКИМИ ГРУППАМИ АВТОМОРФИЗМОВ
}

\section{И. Х. Беккер}

\begin{abstract}
В работе устанавливаются достаточные признаки расщепляемости смешанных абелевых групп с периодическими группами автоморфизмов. Выделяются классы смешанных расщепляющихся абелевых групп с совершенными голоморфами.

Библиографоия: 12 названий.
\end{abstract}

Группы автоморфизмов смешанных абелевых групп мало изучены. В [1], [2] установлены некоторые свойства смешанных абелевых групп с периодическими (в частности, гамильтоновыми) группами автоморфизмов. Ряд задач об автоморфизмах смешанных абелевых групп $G$, их поведении на периодических частях $t G$ и факторгруппах $G / t G$ связан с проблемой расщепляемости груш $G$ [3, проблема 34$]$. Одной из первых работ в этом направлении является [4]. В [5] доказано, что смешанная абелева группа $G$ с периодической нильпотентной группой $\operatorname{Aut} G$ расщепляема. Однако требование нильпотентности - ограничение довольно сильное на периодическую группу Aut $G$ смешанной группы $G$. Периодические части $t G$ таких групп $G$ составляют довольно узкий класс групп - это конечные 2 -групшы вида $t G=\bigoplus_{i=1}^{m} \mathbb{Z}\left(2^{k_{i}}\right)$, где $0<k_{1}<k_{2}<\cdots<k_{m}$. Естественно возникает вопрос: будет ли расщепляемой всякая смешанная абелева группа $G$ с периодической группой Aut $G$ ?

В данной работе установлены достаточные условия расщепляемости смешанных абелевых групा $G$ с периодическими группами Aut $G$. В $\S 1$ рассматриваются свойства таких абелевых групा, приводятся вспомогательные предложения и примеры, доказываются теоремы расщепляемости (теоремы $1.8,1.10$ ). В $§ 2$ изучается вопрос о совершенности голоморфов расщепляюшихся смешанных абелевых групп, части без кручения которых обладают квазиразложениями с сильно неразложимыми сервантными квазислагаемыми.

Под словом "группа" далеепонимаем каждый раз "абелеву группу", кроме еегруппы автоморфизмов и голоморфа. Используемые обозначения стандартны и соответствуют [6] и [7].

\section{$\S 1$. Свойства смешанных групп}

с периодическими группами автоморфизмов

Пусть $G$ - смешанная группа с периодической группой автоморфизмов Aut $G, t G-$ периодическая часть грушы $G, \pi_{t}$ - множество простых чисел, к которому относятся 
примарные компоненты $G_{p}$ группы $t G, t G=\bigoplus_{p \in \pi_{t}} G_{p}$. Групшы $G_{p}$ будем называть также примарными компонентами групшы $G$. Заметим, что смешанная группа $G$ с периодической группой Aut $G$ является редуцированной группой. В противном случае она имеет прямые слагаемые, изоморфные $\mathbb{Q}$ или $\mathbb{Z}\left(p^{\infty}\right)$ для некоторого $p \in \pi_{t}$. Но группа Aut $\mathbb{Q}$ изоморфна мультипликативной группе поля $\mathbb{Q}$, а группа $\operatorname{Aut}\left(\mathbb{Z}\left(p^{\infty}\right)\right)$ изоморфна мультипликативной групе кольца целых $p$-адических $\mathbb{Q}_{p}^{*}$. Следовательно, $G$ - группа редуцированная.

Рассмотрим далее некоторые смешанные групшы.

1. Не существуют копериодические групшы $G$ с периодическими группами Aut $G$.

Действительно, пусть $G$ - копериодическая группа с периодической группой Aut $G$. Тогда $G$ - урегулированная копериодическая группа и Aut $G$ изоморфна группе Aut $(t G)$ $\left[6\right.$, c. 278]. Так как всякая неограниченная группа $G_{p}$ имеет автоморфизмы бесконечного порядка (например, умножение $G_{p}$ на простое число $q \neq p$ ), то все групшы $G_{p}$ ограничены. Периодичность групшы Aut $G$ влечет конечность множества $\pi_{t}$. Следовательно, $t G$ есть сервантная ограниченная подгруппа групшы $G$, и поэтому $t G$ выделяется прямым слагаемым в $G[6$, с. 140$]$. Но это противоречит урегулированности групшы $G$.

2. Всякая квазирасщепляющаяся группа $G$ с периодической группй Aut $G$ - группа расщепляющаяся.

Смешанная группа $G$ назьвается квазирасщепляющейся, если существует такое целое число $n \neq 0$, что $n G \leqslant t G \oplus B \leqslant G$, где $B$ - группа без кручения.

Нетрудно убедиться, что группа $B$ не $p$-делима для любого простого $p, p B \neq B$. Допустим, что группа $G$ имеет неограниченную примарную компоненту $G_{p}$ и для $g \in G$ $n g=a+b=a_{1}+\left(a_{0}+b\right)$, где

$$
a \in t G, \quad a_{1} \in G_{p}, \quad a_{0} \in \underset{\substack{p^{\prime} \in \pi_{t} \\ p^{\prime} \neq p}}{\bigoplus} G_{p^{\prime}}, \quad b \in B .
$$

Отображение $\psi: G \rightarrow G$ вида $\psi g=g-\left(q^{\varphi(n)}-1\right) c_{1} / n$, где $q-$ простое число, $q \neq p$, $(q, n)=1, \varphi(n)-$ функция Эйлера и $q^{\varphi(n)} c_{1}=a_{1}$ есть автоморфизм группы $G$. Так как $G_{p}$ - группа неограниченная, то $\psi$ - автоморфизм бесконечного порядка группы $G$. Следовательно, групп $G$ не имеет неограниченных примарных компонент. Она также не имеет ограниченных бесконечных примарных компонент, так как всякая такая группа разложима в прямую сумму циклических примарных групп и поэтому имеет автоморфизмы бесконечного порядка. Но тогда группа $G$ также имеет такие автоморфизмы, что невозможно. Следовательно, все примарные компоненты групшы $G$ - конечные группы.

Допустим, что $\pi_{t}$ - множество бесконечное. Всякая подгруппа $G_{p}$ выделяется прямым слагаемым в $G[6$, с. 140$]$. Поэтому можно полагать, что существует простое $q \notin \pi_{t}$ взаимно простое с $n,(q, n)=1$. Например, если $\pi_{t}$ есть множество всех простых чисел, то можно $G$ представить в виде $G=A \oplus G^{\prime}$, где $A$ - некоторая периодическая группа, а $G^{\prime}$ - смешанная квазирасшепляющаяся группа с периодической группой Aut $G^{\prime}$ и рассматривать вместо $G$ группу $G^{\prime}$.

Пусть $n G^{\prime} \leqslant t G^{\prime} \oplus B^{\prime} \leqslant G^{\prime}$ и для $g^{\prime} \in G^{\prime}, n g=a+b^{\prime}, b^{\prime} \in B^{\prime}, a \in t G$. Группа $G^{\prime}$ имеет автоморфизм $\omega$ вида $\omega g^{\prime}=g^{\prime}-\left(q^{\varphi(n)}-1\right) c / n$, где $q^{\varphi(n)} c=a$. Очевидно, $\omega-$ автоморфизм бесконечного порядка группы $G^{\prime}$. Следовательно, $\pi_{t}-$ конечное множество и $t G$ выделяется прямым слагаемьм в $G$. 
Лемма 1.1. Если $G$ - смешанная группа с периодической группой Aut $G$, то $G$ обладает такими свойствами:

1) $G$ - әруппа редуиированная;

2) группа $G / t G$ не $p$-делима ни по одному простому числу $p \notin \pi_{t}$;

3) всякая подгруппа $B_{n p}$ базисной подгруппы $B_{p}$ группы $G_{p}$ - группа конечная;

4) $|t G|$ не превосходит мощности континуума;

5) группа $G$ не имеет ограниченных бесконечных примарных компонент $G_{p}$.

ДокАЗАТЕЛЬСтво. 2) Допустим, что факторгрупша $G / t G p$-делима по некоторому простому числу $p \notin \pi_{t}$. Тогда для любого элемента $\bar{g} \in G / t G$ имеем $\bar{g}=p \bar{x}_{0}$ или $\bar{g}=g+t G=p\left(x_{0}+t G\right)$. Отсюда $g=p x_{0}+t_{0}$ для некоторого $t_{0} \in t G$. Так как $p \notin \pi_{t}$, то $t_{0}$ делится на $p, t_{0}=p t_{0}^{\prime}$ и $g=p\left(x_{0}+t_{0}^{\prime}\right)$. Следовательно, сама группа $G-p$-делимая группа, и поэтому отображение $g \rightarrow p g$ является ее автоморфизмом бесконечного порядка, что противоречит условию.

3) Пусть $B_{n p}$ - бесконечная подгруппа, $B_{n p}=\bigoplus_{i \in I}\left\langle g_{i}\right\rangle$, где $o\left(g_{i}\right)=p^{n}$. Так как $B_{n p}$ сервантна в групше $G$ и ограничена, то $B_{n p}$ выделяется прямым слагаемым в $G$, $G=B_{n p} \oplus G^{\prime}$. Группа $B_{n p}$ имеет автоморфизмы бесконечного порядка, поэтому и $G$ имеет такие автоморфизмы.

4) Известно, что если $B_{p}$ - базисная подгруппа редуцированной группы $G_{p}$, то $\left|G_{p}\right| \leqslant\left|B_{p}\right|^{\aleph_{0}}[5$, с. 172$]$. Имеем $\left|B_{p}\right| \leqslant \aleph_{0}$, следовательно, мошность всякой $G_{p}$ группы $t G$ не превосходит мощности континуума. Поэтому мощность $t G$ также не превосходит мощности континуума.

$5)$ Действительно, допустим, что группа $G$ имеет бесконечную ограниченную примарную компоненту $G_{p}$. Так как $G_{p}$ сервантна в $G$, то $G_{p}$ выделяется прямым слагаемым в $G, G=G_{p} \oplus G^{\prime}$. Группа $G_{p}$ разлагается в бесконечную прямую сумму циклических групп [5, с. 107]. Следовательно, $G_{p}$ имеет автоморфизмы бесконечного порядка, поэтому группа $G$ также имеет такие автоморфизмы.

ПРЕДЛОЖЕНИЕ 1.2. а) Для расщепляющейся смешанной группы $G=t G \oplus F$ группа Aut $G$ - периодическая тогда и только тогда, когда $t G$ - группа конечная, a Aut $F$ - әруппа периодическая.

b) Смешанная әруппа $G$ с конечной әруппой Aut $G$ - әруппа расщепляющаяся.

ДокАЗАТЕльство. а) Пусть $G=t G \oplus F$, где $F$ - групша без кручения имеет периодическую групшу автоморфизмов Aut $G$. Тогда $t G=\bigoplus_{p \in \pi_{t}} G_{p}$ такова, что всякая $G_{p}-$ группа конечная, а $\pi_{t}$ есть также множество конечное. Очевидно, что Aut $F$ - група периодическая. Обратно, имеем Aut $G=\Phi \lambda(\operatorname{Aut}(t G) \times \operatorname{Aut} F)$, где $\Phi \cong \operatorname{Hom}(F, t G)$. Следовательно, Aut $G$ - группа периодическая.

b) Если Aut $G$ - групша конечная, то всякая примарная компонента $G_{p}$ групшы $t G-$ группа конечная. Допустив противное, получаем, что группа $G$ имеет бесконечное множество примарных циклических прямых слагаемых. Достаточно учесть, что всякий элемент $a \in G_{p}$ порядка $p$ высоты $h_{p}(a)=k$ можно включить в циклическое прямое слагаемоегрупшы $G$ порядка $p^{k+1}$. Но тогда получаем, что Aut $G$ - групша бесконечная. Множество $\pi_{t}$ также не может быть бесконечным. Следовательно, $t G=\bigoplus_{p \in \pi_{t}} G_{p}$ есть прямая сумма конечного числа конечных примарных компонент. Так как $t G$ сервантна в $G$, то $t G$ выделяется прямым слагаемым в группе $G$. 
СледСтвИе 1.3. Группа автоморфизмов Aut $G$ группы $G$ конечна тогда и только тогда, когда $G=t G \oplus F$, где $F$ - группа без кручения, а группы $t G$, Aut $F u$ $\operatorname{Hom}(F, t G)$ - әруппь конечные.

Следуя [4], автоморфизм $\psi$ группы $t G$ назовем совместным с автоморфизмом $\omega г$ гуппы $\bar{G}=G / t G$, если $\psi$ и $\omega$ индуцируются некоторьм автоморфизмом группы $G$.

Н. Я. Виленкиным поставлен вопрос [3, проблема 34]: следует ли расщепляемость группы $G$ из того, что всякая пара автоморфизмов $\psi, \omega(\psi \in \operatorname{Aut}(t G), \omega \in \operatorname{Aut} \bar{G})$ совместима?

ПРЕДЛОЖЕНИЕ 1.4. Смешанная группа $G$ с периодической группой Aut $G$, у которой всякий автоморфизм $\psi$ ее периодической части совместим с некоторым автоморфизмом группь $\bar{G}$ - группа расщепляющаяся.

Действительно, у такой группы $G$ периодическая часть $t G$ не имеет бесконечньх примарных компонент. Следовательно, все примарные компоненты групшы $G$ - группы конечные. Допустим, что $\pi_{t}-$ множество бесконечное. Тогда групша $t G$ имеет автоморфизм бесконечного порядка, что невозможно. Следовательно, $t G$ - ограниченная сервантная подгруппа группы $G$ и $t G$ выделяется прямым слагаемым в $G$.

СлЕдСТВИЕ 1.5. Смешанная әруппа $G$ с периодической әруппой Aut $G$, у которой всякий автоморфизм ее периодической части $t G$ продолжсается до автоморфизма группь $G$, является расщепляющейся группой.

ПРЕДЛОЖЕНИЕ 1.6. Пусть $G$ - смешанная абелева группа с факторгруппой $G / t G \cong \bigoplus_{i \in I} A_{i}$, где $|I| \geqslant \aleph_{0}, t G=\bigoplus_{p \in \pi_{t}} G_{p}$. Если существует такая инбекиия $\tau: I \rightarrow \pi_{t}$, что $A_{i} \neq \tau(i) A_{i}$, то группа автоморфизмов Aut $G$ не является периодической.

ДокАЗАтЕЛЬСтво. Рассмотрим стабилизатор цепочки $0<t G<G$, изоморфньй группе $\operatorname{Hom}(G / t G, t G)[7$, с. 295]. Имеем

$$
\operatorname{Hom}(G / t G, t G) \cong \operatorname{Hom}\left(\bigoplus_{i \in I} A_{i}, \bigoplus_{p \in \pi_{t}} G_{p}\right) \cong \prod_{i \in I} \operatorname{Hom}\left(A_{i} \bigoplus_{p \in \pi_{t}} G_{p}\right)
$$

Так как $A_{i} \neq \tau(i) A_{i}$, то $\operatorname{Hom}\left(A_{i}, G_{\tau(i)}\right) \neq 0$. Отсюда следует, что $\prod_{i \in I} \operatorname{Hom}\left(A_{i}, G_{\tau(i)}\right)$ содержит гомоморфизмы бесконечного порядка. Следовательно, группа $G$ имеет автоморфизмы бесконечного порядка.

СлЕДСТВИЕ 1.7. Пусть $G$ - смешанная әруппа с периодической әруппой автоморфизмов Aut $G$, факторгруппа $\bar{G}=G / t G$ которой имеет эпиморфный образ $A=\bigoplus_{p \in \pi^{\prime}} A_{p}$, где $A_{p}$ - редучированные $p$-примарные группы. Если $\pi^{\prime \prime}=\pi^{\prime} \cap \pi_{t} \neq \varnothing$ и в случае конечного $\pi^{\prime \prime}=\pi_{t}$ для любого $p \in \pi^{\prime \prime}$ группа $A_{p}$ - неограниченная, то $G$ - расщепляющаяся группа.

Действительно, если допустить, что $\pi^{\prime \prime}$ - множество бесконечное, то группа

$$
\operatorname{Hom}(A, t G) \cong \prod_{p \in \pi^{\prime \prime}} \operatorname{Hom}\left(A_{p}, t G\right)
$$


содержит гомоморфизмы бесконечного порядка. Следовательно, $\pi^{\prime \prime}$ - множество конечное.

Пусть $\pi^{\prime \prime}=\pi_{t}$ и всякая группа $A_{p}\left(p \in \pi^{\prime \prime}\right)$ неограничена, $B=\bigoplus_{n=1}^{\infty} B_{n}-$ базисная подгруппа групшы $A_{p}, G_{p}$ - неограниченная примарная компонента группы $G, B_{p}-\mathrm{ee}$ базисная подгруппа. Имеем $G \stackrel{\pi_{p}}{\rightarrow} A_{p} \stackrel{\chi}{\rightarrow} B$, где $\pi_{p}$ - проекция, а $\chi$ - эпиморфизм группы $A_{p}$ на свою базисную подгруппу $B[6$, с. 179$]$. Далее,

$$
\operatorname{Hom}\left(B, B_{p}\right)=\operatorname{Hom}\left(\bigoplus_{n=1}^{\infty} B_{n}, \bigoplus_{n=1}^{\infty} B_{n p}\right) \cong \prod_{n=1}^{\infty} \operatorname{Hom}\left(B_{n} \bigoplus_{n=1}^{\infty} B_{n p}\right)
$$

и так как $B$ и $B_{p}$ - группы неограниченные, то $\operatorname{Hom}\left(B, B_{p}\right)$ содержит элементы бесконечного порядка. Отсюда следует, что группа $\operatorname{Hom}(G / t G, t G)$ также содержит гомоморфизмы бесконечного порядка. Но тогда групша Aut $G$ не является периодической. Следовательно, в рассматриваемом случае периодическая часть $t G$ группы $G$ ограничена и выделяется прямым слагаемьм в $G$.

Теорема 1.8. Пусть $G$ - смешанная группа с периодической группой автоморфизмов Aut $G$. Если для любого $p \in \pi_{t}$ р-ранг группь $\bar{G}$ бесконечен, то $G-$ расщенляюющаяся группа.

ДокАЗАТЕЛьСтво. Убедимся сначала, что групп $G$ не имеет неограниченных примарных компонент. Пусть $G_{p}$ - неограниченная примарная компонента с базисной подгруппой $B_{p}=\bigoplus_{n=1}^{\infty} B_{n p}$, где $B_{n p}=\bigoplus_{i=1}^{m_{n}}\left\langle g_{n i}\right\rangle$ и $o\left(g_{n i}\right)=p^{n}, 1 \leqslant m_{n}<\infty$ (лемма 1.1). Существуют гомоморфизмы группы $\bar{G}=G / t G$ в групшы $B_{n p}(n=1,2, \ldots)$. Следовательно, для любого $n$ существуют также гомоморфизмы $\omega_{n}$ групшы $\bar{G}$ в $B_{n p}$, $\omega_{n}: \bar{G} \rightarrow B_{n p}$. Тогда существует такой единственный гомоморфизм $\varphi: \bar{G} \rightarrow \prod_{n=1}^{\infty} B_{n p}$, что все диаграммы вида

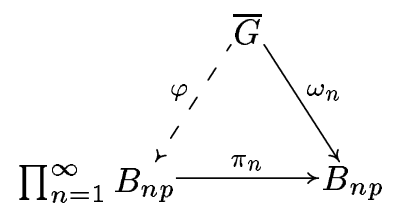

коммутативны, где $\pi_{n}$ проекции $[6$, с. 53]. Гомоморфизм $\varphi$ строится следующим образом: для $\bar{g} \in \bar{G}$ полагаем $\varphi \bar{g}=b$, где $b$ - единственньй элемент из $\prod_{n=1}^{\infty} B_{n p}$, для которого $\pi_{n} b=\omega_{n} \bar{g}$ и $\pi_{n} \varphi=\omega_{n}$ для любого $n \geqslant 1$. Если гомоморфизмы $\omega_{n}$ таковы, что $\omega_{n} \bar{g}=0$ почти для всех $n$ (т.е. лишш для конечного числа индексов $n \omega_{n} \bar{g} \neq 0$ ), то получаем некоторьй гомоморфизм $\eta$ группы $\bar{G}$ в базисную подгруппу $B_{p}$ группы $G_{p}$, $\eta: \bar{G} \rightarrow B_{p}$. Так как $p$-ранг группы $\bar{G}$ бесконечен и $G_{p}-$ группа неограниченная, то такие гомоморфизмы $\eta$ можно строить так, чтобы порядки элементов $\omega_{n} \bar{g}$ не были ограничены в совокупности. В таких случаях получаем, что порядок гомоморфизмов $\eta$ грушы $\bar{G}$ в $G_{p}$ бесконечен. Рассмотрим теперь стабилизатор цепочки $0<t G<G$, изоморфный группе $\operatorname{Hom}(G / t G, t G)$. Он является нормальной подгруппой группы Aut $G[7, \mathrm{c} .295]$. Но по условию Aut $G$ - группа периодическая, следовательно, группа $G$ не содержит неограниченных примарных компонент. 
Покажем теперь, что $\pi_{t}-$ множество конечное. Допустим противное и рассмотрим диаграммы вида

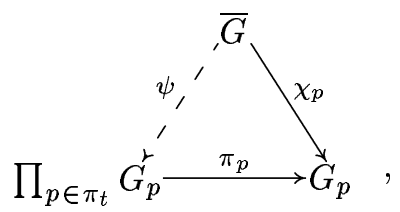

где $\chi_{p}$ - гомоморфизм не $p$-делимой групшы $\bar{G}$ в $G_{p}, \chi_{p}: \bar{G} \rightarrow \bar{G} / p \bar{G} \rightarrow G_{p}, \pi_{p}-$ проекции. Существует единственньй гомоморфизм $\psi$, замыкающий эти диаграммы до коммутативных [6, с. 53]. Пусть $\chi_{p}$ таковы, что для любого $\bar{g} \in \bar{G}$ и почти для всех $p \in \pi_{t}$ $\chi_{p} \bar{g}=0$. Тогда получаем замыкающий гомоморфизм $\bar{\psi}: \bar{G} \rightarrow t G=\bigoplus_{p \in \pi_{t}} G_{p}$. Учитывая теперь бесконечность множества $\pi_{t}$ и $p$-рангов группы $\bar{G}$ для любого $p \in \pi_{t}$, получаем, что среди гомоморфизмов $\bar{\psi}: \bar{G} \rightarrow t G$ есть гомоморфизмы бесконечного порядка. Следовательно, стабилизатор цепочки $0<t G<G$, изоморфный группе $\operatorname{Hom}(G / t G, t G)$ содержит автоморфизмы бесконечного порядка, что невозможно. Доказано, что $\pi_{t}-$ множество конечное.

Итак, периодическая часть $t G$ смешанной групшы $G$ не содержит неограниченных примарных компонент и $t G=\bigoplus_{i=1}^{k} G_{p_{i}}(1 \leqslant k<\infty)$. Следовательно, $t G-$ ограниченная сервантная подгруппа группы $G$ и $t G$ выделяется прямым слагаемым в $G$. Теорема доказана.

СлЕДСТВИЕ 1.9. Если $G$ - смешанная группа с периодической группой автоморфизмов Aut $G$ и $\bar{G}=G / t G$ имеет бесконечный $p$-ранг, $p \in \pi_{t}$, то $p$-компонента $G_{p}$ - группа ограниченная.

Пусть далее $\Pi(\bar{G})=\{p \mid p \bar{G} \neq \bar{G}\}, \Pi(\bar{G}) \cap \pi_{t}=\varnothing, B_{p}^{\prime}-p$-базисная подгрупшагруппы $G, B_{p}^{\prime}=B_{0 p} \oplus\left(\bigoplus_{n=1}^{\infty} B_{n p}\right)$, где $B_{0 p}=\bigoplus \mathfrak{N}_{p} \mathbb{Z}, B_{n p}$ - прямая сумма циклических групा порядка $p^{n}(n \geqslant 1)$. Для всякого $n$ групша $B_{n p}$ сервантна в $G$. Если Aut $G$ - группа периодическая, то $B_{n p}$ - группа конечная (лемма 1.1), $B_{n p}=\bigoplus_{i=1}^{m_{n}}\left\langle g_{n i}\right\rangle$, где $o\left(g_{n i}\right)=p^{n}$, $1 \leqslant m_{n}<\infty$. Рассмотрим цепочку подгрупा $0<\bar{B}_{p}<G$, где $\bar{B}_{p}=\bigoplus_{n=1}^{\infty} B_{n p}$. Убедимся, что $G / \bar{B}_{p}=\widehat{G}$ - группа не $p$-делимая. Допустим противное, пусть уравнение $b_{0}+\bar{B}_{p}=p^{k}\left(x+\bar{B}_{p}\right)$, где $0 \neq b_{0} \in B_{0 p}$ разрешимо в $\widehat{G}$ для всякого $k \geqslant 1$. Отсюда $b_{0}=p^{k} x_{0}+\bar{b}$ для некоторых $x_{0} \in G, \bar{b} \in \bar{B}_{p}$ и $p^{m} b_{0}=p^{k+m} x_{0}$, где $p^{m}=o(\bar{b})$. Так как $B_{0 p} p$-сервантна в $G[6$, c. 163$]$, то $x_{0} \in B_{0 p}$. Тогда из $p^{m}\left(b_{0}-p^{k} x_{0}\right)=0$ следует, что $b_{0}=p^{k} x_{0}$ и $B_{0 p}-p$-делимая группа. Следовательно, группа $\widehat{G}$ не является $p$-делимой.

Теорема 1.10. Пусть $G$ - смешанная группа с периодической группой автомор$\oint$ фимов Aut $G$, у которой $\Pi(\bar{G}) \cap \pi_{t}=\varnothing$. Если для любого $p \in \pi_{t}$ ранг без кручения $\mathfrak{N}_{p}$ р-базисной подгруппь группь $G$ бесконечен, то $G$ - группа расщепляющаяся.

ДокАЗАТЕЛЬСтво. Для того чтобы убедиться, что группа $G$ не содержит неограниченных примарных компонент, достаточно рассмотреть стабилизатор цепочки $0<$ $\bar{B}_{p}<G$ и учесть, что $\mathfrak{N}_{p}-$ бесконечньй кардинал.

Пусть $\pi_{t}-$ множество бесконечное, $\widehat{B}=\bigoplus_{p \in \pi_{t}} t B_{p}^{\prime}$ и $\widetilde{G}=G / \widehat{B}$. Для любого $p \in \pi_{t}$ группа $\widetilde{G}$ не $p$-делима. Допустим противное, пусть $\widetilde{G} p_{1}$-делимая групша для некоторого $p_{1} \in \pi_{t}$. Тогда для $b_{0} \in B_{0 p_{1}}$ и любого $k \geqslant 1$ в $\widetilde{G}$ разрешимо уравнение $b_{0}+\widehat{B}=p_{1}^{k}(x+\widehat{B})$. Отсюда $b_{0}=p_{1} x_{0}+\widehat{b}$ для некоторых $x_{0} \in G$ и $\widehat{b} \in \widehat{B}$. Пусть $\widehat{b}=b_{1}+b_{2}$, где $b_{1} \in t B_{p_{1}}, b_{2} \in \bigoplus_{p \neq p_{1}} t B_{p}^{\prime}, o\left(b_{1}\right)=p_{1}^{s}, o\left(b_{2}\right)=m$, причем $\left(m, p_{1}\right)=1$. 
Имеем $p_{1}^{s} b_{0}=p_{1}^{s}\left(p_{1}^{k} x_{0}+b_{2}\right)$ и так как $B_{0 p_{1}}-p_{1}$-сервантная подгрупша групшы $G$, то $p_{1}^{k} x_{0}+b_{2} \in B_{0 p_{1}}$. Но $b_{2}=p_{1}^{k} b_{2}^{\prime}$ для некоторого $b_{2}^{\prime} \in \bigoplus_{p \neq p_{1}} t B_{p}^{\prime}$, тогда $b_{0}=p_{1}^{k}\left(x_{0}+b_{2}^{\prime}\right)$ и $B_{0 p_{1}}-p_{1}$-делимая группа, что невозможно. Следовательно, групша $\widetilde{G}$ не $p$-делима ни на одно $p \in \pi_{t}$. Рассмотрим теперь стабилизатор цепочки $0<\widehat{B}<G$, т.е. группу $\operatorname{Hom}(G / \widehat{B}, \widehat{B})$. Для каждой $t B_{p}$ группа $\operatorname{Hom}\left(\widetilde{G}, t B_{p}\right) \neq 0$. Существуют такие гомоморфизмы $\tau: \widetilde{G} \rightarrow \prod_{p \in \pi_{t}} t B_{p}$, что все диаграммы

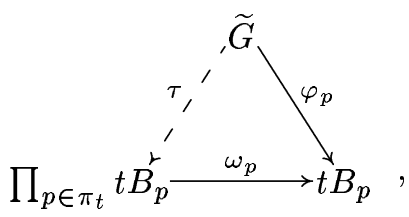

где $\varphi_{p} \in \operatorname{Hom}\left(\widetilde{G}, t B_{p}\right), \omega_{n}-$ проекции, коммутативны [6, с. 53]. В случае бесконечного $\pi_{t}$, учитывая, что всякий кардинал $\mathfrak{N}_{p}$ бесконечен, можно строить замыкающие гомоморфизмы $\tau^{\prime}: \widetilde{G} \rightarrow \bigoplus_{p \in \pi_{t}} t B_{p}<\prod_{p \in \pi_{t}} t B_{p}$ бесконечного порядка. Для этого достаточно положить, что для любого $\widetilde{g} \varphi_{p} \widetilde{g}=0$ почти для всех $p \in \pi_{t}$. Но тогда получаем, что группа Aut $G$ содержит автоморфизмы бесконечного порядка. Следовательно, $\pi_{t}-$ множество конечное и так как все компоненты $G_{p}$ ограничены, то $t G$ - группа ограниченная и прямое слагаемое групшы $G, G=t G \oplus F$, где $F$ - групша без кручения.

\section{§2. Смешанные абелевы группы с совершенными голоморфами}

Пусть $G$ - смешанная группа, $\Gamma(G)$ - ее голоморф, т.е. полупрямое расширение группы Aut $G$ с помошью групшы $G$. Всякий элемент группы $\Gamma(G)$ рассматриваем как паpy $(g, \varphi), g \in G, \varphi \in \operatorname{Aut} G$. Груповая операция определена в $\Gamma(G)$ по правилу: если $(g, \varphi)(a, \psi) \in \Gamma(G)$, то $(g, \varphi)+(a, \psi)=(g+\varphi a, \varphi \psi)$. Элементы групшы $\Gamma(G)$ вида $(g, \varepsilon)$, где $\varepsilon$ - единица группы Aut $G$, образуют нормальную подгруппу, изоморфную группе $G$ (отождествляем ее с $G$ ), а элементы вида $(0, \varphi)$, где 0 - нулевой элемент группы $G$, образуют подгруппу, изоморфную $\operatorname{Aut} G$ (отождествляем еe с Aut $G$ ). Итак, $\Gamma(G)=G \dashv$ Aut $G, G \cap \operatorname{Aut} G=(0, \varepsilon)$. Заметим, что если $N$ - нормальная подгруппа группы $\Gamma(G)$ и $N_{1}, \Psi_{1}$ - соответственно множества всех первых, вторых компонент ее элементов, то $N_{1}$ - характеристическая подгруппа групшы $G$, а $\Psi_{1}$ - нормальная подгруппа групшы Aut $G$. Группа $G$ является максимальной нормальной абелевой подгруппой групшы $\Gamma(G)$ и ее централизатор $C_{\Gamma}(G)=G$. Групу $G$ назовем голоморфно разложимой, если из того, что $(g, \varphi) \in G^{\theta}$, где $\theta$ - автоморфизм ее голоморфа, следует $(g, \varepsilon)$, $(0, \varphi) \in G^{\theta}$. Такими группами являются, например, группы с нулевыми 2-компонентами и другие [8].

Пусть $\operatorname{Aut}(\Gamma), \operatorname{Aut}^{\prime}(\Gamma), \operatorname{Aut}_{G}(\Gamma)$ - соответственно группа всех автоморфизмов, группа всех внутренних автоморфизмов, группа всех автоморфизмов, отображающих группу $G$ на себя, группы $\Gamma(G)$. Имеем $\operatorname{Aut}^{\prime}(\Gamma) \leqslant \operatorname{Aut}_{G}(\Gamma) \leqslant \operatorname{Aut}(\Gamma), \operatorname{Aut}^{\prime}(\Gamma) \unlhd \operatorname{Aut}(\Gamma)$ и $\operatorname{Aut}_{G}(\Gamma) / \operatorname{Aut}^{\prime}(\Gamma)$ изоморфна первой группе когомологии групшы Aut $G$ над группой $G$, $\operatorname{Aut}_{G}(\Gamma) / \operatorname{Aut}^{\prime}(\Gamma) \cong H^{1}($ Aut $G, G)$.

Рассмотрим вопрос о совершенности голоморфа смешанной групшы $G$ с периодической группой Aut $G$. Заметим, что если $\Gamma(G)$ - группа с центром $C(\Gamma)$, то $C(\Gamma)$ является 2-группой. Для решения вопроса о совпадении групп $\operatorname{Aut}^{\prime}(\Gamma)=\operatorname{Aut}_{G}(\Gamma)$ (т.е. вопроса о характеристичности группы $G$ в группе $\Gamma(G))$ воспользуемся таким свойством голоморфа абелевой группы. 
ЛЕмма 2.1 [8, следствие 1]. Пусть $G$ - голоморфно разложимая әруппа. Если $\Gamma(G)$ имеет такой автоморфизм $\theta$, что $G^{\theta} \neq G$ и $G_{1}, \Phi_{1}$ - соответственно множества всех первых, вторых компонентов әлементов $G^{\theta}$, то

1) $G^{\theta}=G_{1} \oplus \Phi_{1}, G_{1} \neq\langle(0, \varepsilon)\rangle, \Phi_{1} \neq\langle(0, \varepsilon)\rangle$;

2) $G_{1}$ - характеристическая подгруппа и прямое слагаемое группь $G, G=$ $G_{1} \oplus G_{2}$

3) $\Phi_{1} \cong \operatorname{Hom}\left(G_{2}, G_{1}\right)$.

ПРЕДЛОЖЕНИЕ 2.2. Всякая голоморфно разложимая смешанная группа с периодической әруппой автоморфизмов Aut $G$ характеристична в своем голоморфе.

ДокАЗАТЕЛЬСтво. Пусть $\theta$ - такой автоморфизм группы $\Gamma(G)$, что $G^{\theta} \neq G$. Тогда по лемме 2.1 получаем разложения $G^{\theta}=G_{1} \oplus \Phi_{1}, G=G_{1} \oplus G_{2}$ и $G_{1}$ - характеристическая подгруппа групшы $G$. Пусть $\pi_{i}\left(t G_{i}\right)$ - множество простых чисел, к которому относится периодическая часть $t G_{i}$ группы $G_{i}(i=1,2)$. Убедимся, что $t G \leqslant G_{1}$, т.е. $\pi_{2}\left(t G_{2}\right)=\varnothing$. Действительно, пусть $p \in \pi_{1}\left(t G_{1}\right) \cap \pi_{2}\left(t G_{2}\right)$. Тогда $G_{1}$ и $G_{2}-$ смешанные редуцированные группы. Они имеют циклические прямые слагаемые порядка $p^{\alpha}$ $(\alpha \geqslant 1)$, поэтому $\operatorname{Hom}\left(G_{1}, G_{2}\right) \neq 0$. Это противоречит характеристичности $G_{1}$ в $G_{2}$. Допустим, что $\pi_{1}\left(t G_{1}\right) \cap \pi_{2}\left(t G_{2}\right)=\varnothing$ и $p \in \pi_{2}\left(t G_{2}\right)$. Тогда $G_{2}$ содержит примарную компоненту, каждый элемент которой неподвижен относительно всех автоморфизмов из $\Phi_{1}$. Следовательно, централизатор $C_{\Gamma}\left(G^{\theta}\right)$ групшы $G^{\theta}$ относительно $\Gamma(G)$ не совпадает c $G^{\theta}$, т.е. $G^{\theta}$ не является максимальной нормальной абелевой подгруппой группы $\Gamma(G)$, что невозможно. Отсюда следует $\pi_{2}\left(t G_{2}\right)=\varnothing, t G \leqslant G_{1}$ и $\Phi_{1}, G_{2}-$ групшы без кручения. Если допустить, что $p G_{2} \neq G_{2}$ хотя бы для одного $p \in \pi_{1}\left(t G_{1}\right)=\pi_{t}$, то $\operatorname{Hom}\left(G_{2}, G_{1}\right)$ содержит гомоморфизмы порядка $p^{\alpha}(\alpha \geqslant 1)$, что невозможно. Если $G_{2} p$-делима для некоторого $p \in \pi_{t}$, то $G_{2}$ имеет автоморфизмы бесконечного порядка, что противоречит условию.

Пусть, далее, $G$ - смешанная расщепляющая группа с периодической группой автоморфизмов Aut $G, G=t G \oplus F$. Согласно предложению $1.2 t G$ - группа конечная, а $F-$ группа без кручения с периодической группой Aut $F$. Рассмотрим теперь вопрос: для каких групп $G$ групшы $\operatorname{Aut}_{G}(\Gamma)$ и $\operatorname{Aut}^{\prime}(\Gamma)$ совпадают (т.е. $\left.H^{1}(\operatorname{Aut} G, G)=0\right)$ ? Пусть $t G=\bigoplus_{i=1}^{k} G_{p_{i}}$, если 2-компонента групшы $t G$ нулевая, то $H^{1}$ (Aut $\left.t G, t G\right)=0[9]$. Пусть $G_{2} \neq 0, G_{2}=\bigoplus_{i=1}^{m} \mathbb{Z}\left(n_{i}\right)$, где $n_{i}=2^{k_{i}}$ и $n_{1} \geqslant n_{2} \geqslant \cdots \geqslant n_{m}$. Согласно [10] $H^{1}\left(\right.$ Aut $\left.G_{2}, G_{2}\right)=0$ тогда и только тогда, когда $n_{1} / 2=n_{2}=\cdots=n_{m}$ или $n_{1}=n_{2}<4 n_{3}=4 n_{4}=\cdots=4 n_{m}$. Следовательно, $H^{1}($ Aut $t G, t G)=0$ тогда и только тогда, когда $H^{1}\left(\right.$ Aut $\left.G_{2}, G_{2}\right)=0$ [9] и вычисление групшы $H^{1}($ Aut $G, G)$ сводится к вычислению групшы $H^{1}$ (Aut $\left.F, F\right)$. При изучении этой групшы потребуются некоторые понятия, введенные в [9]. Группа $F$ является $F N$-группой (т.е. группой без нильпотентных эндоморфизмов) [7, с. 315]. Подгруппа $A$ группы $F$ назьвается квазиравной $F, F \approx A$, если для некоторого натурального $m m F \leqslant A$. Если $\left\{F_{i}\right\}_{i \in I}$ - такое семейство подгрупп группы $F$, что $F \approx \bigoplus_{i \in I} F_{i}\left(I-\right.$ произвольное множество индексов), то $\bigoplus_{i \in I} F_{i}$ назьвается квазиразложсением группы $F$, а $F_{i}$ - ее квазислагаемым. Если $F$ имеет лишь тривиальные квазиразложения, то $F$ назьвается сильно неразложимой. Оказывается, что всякая конечная група автоморфизмов $\Phi \neq\langle\varepsilon\rangle, \Phi \leqslant$ Aut $F$, содержащая нерегулярные автоморфизмы, индуцирует некоторое квазиразложение группы $F$ вида 
$F \approx \bigoplus_{i=1}^{s} F_{i}$, где каждое квазислагаемое $F_{i}$ сервантно и вполне характеристично в $F[9]$.

Пусть группа $F$ обладает квазиразложением $F \approx \bigoplus_{i \in I} F_{i}$, где каждое квазислагаемое $F_{i}$ сильно неразложимо и сервантно в $F$. Для такой группы $F$ без кручения с периодической группой Aut $F$ справедлив аналог теоремы Холлета-Хирша о строении конечной групшы автоморфизмов Aut $B$ абелевой групшы $B$ без кручения $[7$, c. 318]. B таком случае группа Aut $F$ и всякая ее подгруппа $\Phi \neq\langle\varepsilon\rangle$ являются подпрямыми произведениями групп, изоморфных $\mathbb{Z}(n)(n=2,3,4,6), Q_{8}-$ групе кватернионов, $D C_{12}-$ дициклической группе порядка 12 или $B T_{24}$ - бинарной групе тетраэдра порядка 24 [9]. Если $F \approx \bigoplus_{i \in I} F_{i}$ - квазиразложение групшы $F$ без кручения с сильно неразложимыми сервантньми квазислагаемьми $F_{i}$ конечного ранга, то $\bigoplus_{i \in I} F_{i}$ называется $n о л н ы м ~$ квазиразложсением группы $F[11]$.

ПРЕДЛОЖЕНИЕ 2.3. Пусть $F-$ сильно неразложимая группа без кручения с периодической әруппой Aut $F$.

Голомор $\oint \Gamma(F)$ группы $F$ совершенен тогда и только тогда, когда Aut $F$ изоморфна $\mathbb{Z}(6), D C_{12}$ или $B T_{24}$.

ДокАЗАтЕльство. Согласно лемме 2.1 группа $F$ характеристична в своем голоморфе. Так как $F$ - группа без кручения, то $\Gamma(F)$ - групша без центра. Пусть $\Gamma(F)$ - группа совершенная. Заметим, что всякий автоморфизм $\varphi \neq \varepsilon$ грушы $F$ регулярен. В противном случае $F$ имеет нетривиальные квазиразложения. Отсюда следует, что Aut $F$ конечна и изоморфна одной из групп $\mathbb{Z}(n)(n=2,4,6), Q_{8}, D C_{12}$ или $B T_{24}[9]$. Если допустить, что Aut $F$ изоморфна $\mathbb{Z}(2), \mathbb{Z}(4)$ или $Q_{8}$, то получаем, что $H^{1}$ (Aut $\left.F, F\right) \neq 0$, т.е. $\operatorname{Aut}^{\prime}(F) \neq \operatorname{Aut}_{G}(F)[9]$. Следовательно, совершенность $\Gamma(F)$ влечет изоморфизм группы Aut $F$ одной из групп $\mathbb{Z}(6), D C_{12}$ или $B T_{24}$. Обратно, пусть Aut $F$ изоморфна одной из этих трех групп. В каждом из этих случаев группа $F$ имеет регулярньй автоморфизм нечетного порядка, поэтому $H^{1}(\mathrm{Aut} F, F)=0$ [9]. Следовательно, в каждом из этих случаев голоморф $\Gamma(F)$ совершенен.

СлЕДСТВИЕ 2.4. Пусть $F$ - әруппа без кручения с периодической группой Aut $F$ и $F \approx \bigoplus_{i \in I} F_{i}$ - ее квазиразложсение с сильно неразложсимыми сервантными квазислагаемыми $F_{i}$.

Голомор $\oint\left(F_{i}\right)$ квазислагаемого $F_{i}$ конечного ранга совершенен тогда и только тогда, когда Aut $F_{i}$ изоморфна одной из групп $\mathbb{Z}(6), D C_{12}$ или $B T_{24}$.

Достаточно учесть, что сильно неразложимое $F_{i}$ конечного ранга групшы $F$ с периодической группой Aut $F$ имеет конечную группу Aut $F_{i}[9]$. Если допустить, что такое квазислагаемое $F_{i}$ имеет автоморфизм $\psi$ бесконечного порядка, то некоторая его степень $\psi^{m}(m \geqslant 1)$ может быть продолжена до автоморфизма самой групшы $F$ [11].

Tеорема 2.5. Пусть $G=t G \oplus F-$ смешанная группа с $G_{2}=0$ и периодической әруппой Aut $G$, часть без кручения $F$ которой обладает квазиразлохсением $F \approx \bigoplus_{i \in I} F_{i}$ с сильно неразложсимыми сервантными $F_{i}$ и периодическими Aut $F_{i}$.

1) Если голоморф группы $G$ совершенен, то каждая группа $F_{i}$ имеет совершенным голоморф.

2) $\operatorname{Ecли~} \mathbb{Z}(3)<\Phi_{i}=$ Aut $\left.F\right|_{F_{i}}$ для всякого $i \in I$, то голоморф $\Gamma(G)$ группь $G$ совершенен. 
ДокАЗАТЕЛьство. 1) Заметим, что каждая $F_{i}$ характеристична в $F[9]$ и так как $F_{i}$ сильно неразложима, то по лемме $2.1 F_{i}$ характеристична в своем голоморфе. Каждьй ее автоморфизм $\varphi \neq \varepsilon$ регулярен и Aut $F_{i}$ изоморфна одной из групा $\mathbb{Z}(n)(n=2,4,6)$, $Q_{8}, D C_{12}$ или $B T_{24}$.

Пусть $\Gamma(G)$ - группа совершенная, тогда $H^{1}($ Aut $F, F)=0$ [12]. Отсюда следует, что и каждая группа $H^{1}\left(\Phi_{i}, F_{i}\right)=0$, где $\Phi_{i}=\left.\operatorname{Aut} F\right|_{F_{i}}, \Phi_{i} \leqslant$ Aut $F_{i}$ [9]. Следовательно, каждая из груп $\Phi_{i}$ содержит элементы порядка 3 . Если допустить противное, то $\Phi_{i}$ есть 2-группа, и тогда $H^{1}\left(\Phi_{i}, F_{i}\right) \neq 0$ [9]. Доказано, что всякая група $H^{1}\left(\right.$ Aut $\left.F_{i}, F_{i}\right)=0$ и каждая Aut $F_{i}$ изоморфна $\mathbb{Z}(6), D C_{12}$ или $B T_{24}$. Согласно предложению 2.3 получаем, что всякая группа $F_{i}$ имеет совершенньй голоморф.

2) Группа $G$, удовлетворяющая условию теоремы, характеристична в своем голоморфе (предложение 2.2). Убедимся, что групша $H^{1}($ Aut $F, F)=0$. Пусть каждая $\Phi_{i}$ содержит элементыпорядка 3 . Следовательно, $\Phi_{i}$ содержит регулярные автоморфизмы нечетного порядка группы $F_{i}$, поэтому $H^{1}\left(\Phi_{i}, F_{i}\right)=0[9]$. Тогда

$$
H^{1}\left(\prod_{i \in I} \Phi_{i}, \bigoplus_{i \in I} F_{i}\right)=0
$$

Отсюда следует, что групша $H^{1}(\mathrm{Aut} F, F)$ также нулевая [9], но тогда и $H^{1}(\mathrm{Aut} G, G)=0$ [12]. Так как $G$ не содержит элементов порядка 2 , то $\Gamma(G)$ - группа без центра. Доказано, что голоморф групшы $G$ совершенен.

СлЕдСТВИЕ 2.7. Пусть $G$ - расщепляющаяся смешанная группа с нулевой 2-компонентой и периодической группой Aut $G$, удовлетворяющая одному из условий:

1) F разложима в прямую сумму сильно неразложимых групп;

2) всякий автоморфизм каждого квазислагаемого $F_{i}$ квазиразложсения $F \approx$ $\bigoplus_{i \in I} F_{i}$ с сильно неразлохимыми сервантными $F_{i}$ может быть продолжен до автоморфизма группы $F$.

Голомор $Г(G)$ группы $G$ совершенен тогда и только тогда, когда каждая группа $\Gamma\left(F_{i}\right)$ совершенна.

СлЕДСТВИЕ 2.8. Пусть $G$ - расщепляющаяся смешанная группа с нулевой 2-компонентой и периодической группой Aut $G$, удовлетворяющая одному из условий:

1) $F$ имеет полное квазиразложение, $F \approx \bigoplus_{i \in I} F_{i}$;

2) $G$ - группа конечного ранга;

3) Aut $F$ - группа конечная и $F \approx \bigoplus_{i=1}^{m} F_{i}$ - квазиразложсение, индуцированное әруппой Aut $F$.

Группа $G$ имеет совершенный голоморф тогда и только тогда, когда каждая әруппа $\Phi_{i}=$ Aut $\left.F\right|_{F_{i}}$ изоморфна $\mathbb{Z}(6), D C_{12}$ или $B T_{24}$.

ЗАмечАниЕ. Если группа без кручения $F$ имеет периодическую группу автоморфизмов Aut $F$, то Aut $F$ - групша разрешимая [9]. Будет ли разрешимой периодическая группа автоморфизмов Aut $G$ расшепляющейся смешанной групшы $G$ ?

Учитывая [2], получаем полньй ответ на этот вопрос. 
Периодическая групша автоморфизмов Aut $G$ расщепляющейся смешанной группы $G$ разрешима тогда и только тогда, когда каждая ее примарная компонента

$$
G_{p}=\bigoplus_{i=1}^{n} \bigoplus_{m_{i p}} \mathbb{Z}\left(p^{k_{i p}}\right)
$$

где $0<k_{1 p}<k_{2 p}<\cdots<k_{n p}, n$ не зависит от $p$ и $m_{i p}=0,1$ или 2 при $p=2,3 ; m_{i p}=0$ или 1 при $p \geqslant 5$.

Томский государственный университет

\section{СПИСОК ЦИТИРОВАННОЙ ЛИТЕРАТУРЫ}

[1] Шляфер А. З. Об абелевых группах с периодическими группами автоморфизмов // Абелевы группы и модули. Вьп. 6. Томск, 1986. С. 165-177.

[2] Шляфер А. З. О разрешимости групп автоморфизмов абелевых групп // Абелевы группы и модули. Вып. 7. Томск, 1987. С. 148-155.

[3] Fuchs L. Abelian groups. Budapest: Acad. Kiado, 1966.

[4] Мишина А. П. Некоторые условия расщепления смешанных абелевых групп // Укр. матем. ж. 1951. T. 3. C. $218-232$.

[5] Шляфер А. З. Периодические группы автоморфизмов абелевых групп и модулей. Дисс. . . . к. ф.-м.н. Томск: ТГУ, 1986.

[6] Фукс Л. Бесконечные абелевы группы. Т. 1. М.: Мир, 1974.

[7] Фукс Л. Бесконечные абелевы группы. Т. 2. М.: Мир, 1977.

[8] Беккер И. Х. О голоморфах нередуцированных абелевых групп // Изв. вузов. Матем. 1968. № 8 . C. $3-10$.

[9] Беккер И. Х. Об абелевых группах без кручения с периодическими группами автоморфизмов // Изв. вузов. Матем. 1986. № 2. С. 3-12.

[10] Mills W. H. The automorphisms of the holomorph of a finite Abelian group // Trans. Amer. Math. Soc. 1957. V. 85. № 1. P. 1-34.

[11] Кожухов С.Ф. Абелевы группы без нильпотентных эндоморфизмов // Абелевы группы и модули. Томск, 1979. С. 87-94.

[12] Беккер И. Х. О группах скрещенных голоморфизмов групп автоморфизмов абелевых групп без кручения // Изв. вузов. Матем. 1973. № 7. С. 3-11. 